\title{
Hyperthermal Chaotic Photodesorption of Xenon from Alumina-Supported Silver Nanoparticles: Plasmonic Coupling and Plasmon-Induced Desorption
}

\author{
Kazuo Watanabe, ${ }^{1}$ Ki Hyun Kim, ${ }^{1}$ Dietrich Menzel, ${ }^{1,2}$ and Hans-Joachim Freund ${ }^{1}$ \\ ${ }^{1}$ Fritz-Haber-Institut der Max-Planck-Gesellschaft, Faradayweg 4-6, 14195 Berlin, Germany \\ ${ }^{2}$ Physik-Department E20, Technische Universität München, 85748 Garching, Germany
}

(Received 9 March 2007; published 26 November 2007)

\begin{abstract}
Excitation of Xe monolayers on alumina-supported silver nanoparticles (AgNPs) by laser light in the $(1,0)$ Mie plasmon resonance can lead to desorption of Xe atoms with hyperthermal energy and chaotic time structure. The chaotic behavior is most likely due to plasmonic coupling between AgNPs. We argue that the desorption is induced by direct energy transfer to the adsorbate from the Pauli repulsion of the collectively oscillating electrons of the plasmon at the surface. A simple model calculation shows that this is possible. A connection between both effects appears likely.
\end{abstract}

Metal nanoparticles (MNPs) in the size range 1 to $20 \mathrm{~nm}$ have special properties, not only in catalysis and other near ground state surface properties, but also with respect to electronic excitations and, consequently, to photoninduced surface reactions [1,2]. Important factors are the confinement of electronic and phononic excitations in the particles, which may lead to quite strong changes of their lifetimes. For MNPs containing quasifree electrons the most drastic change in the excitation spectrum is the existence of the so-called Mie plasmon [3]. These two effects can concur, leading to special properties of MNPs for photoreactions [2]. Drastic increases in photoreaction cross sections by excitation in the plasmon resonance have been observed [4-8], with the first work [4] reporting the nonthermal evaporation of $\mathrm{Na}$ atoms from NaNPs in their plasmon range. However, it appears that - with the possible exception on ref. [4] — no clear indication of a unique action of the plasmon itself has been seen; all cases reported, e.g., of photodesorption, may be understood either in terms of cross section enhancement due to the plasmoninduced field enhancement without change of the nonthermal desorption mechanism [5-7], or by strongly enhanced thermal effects [8]. Nonthermal photodesorption usually occurs through the transient excitation of a repulsive adsorbate state. In the energy range concerned (about 2 to $4 \mathrm{eV}$ ) the main mechanism goes via transient negative ions (TNI mechanism): absorption of the light by the metal substrate creates hot electrons; the latter can form transient negative ions of the adsorbed species with shifted potentials which leads to momentum transfer and ultimatelyafter backtransfer of the excited electron to the substrateto desorption [9]. The events appear to be essentially the same in and off the plasmon resonance. The Mie plasmon is known to have a very short lifetime (of around $10 \mathrm{fs}$ or less) [10] and partly decays into electron-hole pairs which can enter this mechanism. Besides these nonthermal processes, confinement of the large energy input into the MNPs in the plasmon resonance can also lead to strong thermal events (diffusion, shape changes, desorption), even in the MNPs themselves [11].

Here we report a case of nonthermal desorption where the plasmon is not just a means to drastically increase the energy input into the MNPs, but where it appears to be desorption-active itself. The initial idea of our investigation was to select a case in which no desorption via transient negative ions is possible, so that such a mechanism should be easy to discern. This is indeed the case. However, the situation turned out not to be that simplewe also found strange chaotic behavior in desorption. As will be explained we believe that these two characteristics - direct plasmon-induced desorption, and chaotic response - are intimately connected.

The selected MNP system was the very well characterized case of silver nanoparticles (AgNPs) supported on thin alumina films on $\mathrm{NiAl}(110)$ [12]. In order to identify plasmon-induced desorption we studied monolayers of Xe physisorbed on the AgNPs. The TNI mechanism will not work here because no affinity level is stable on Xe overlayers on metals [13], and desorption via direct excitation of the adatoms $[14,15]$ is not possible with UV photons due to the large HOMO-LUMO gap $(\sim 9 \mathrm{eV})$ of the Xe layer [16]. In fact, no nonthermal photodesorption of rare gas atoms from bulk metal surfaces has been reported in the uv range so far. Nevertheless, we do observe nonthermal desorption of Xe from AgNP surfaces. This finding as well as the connected very peculiar behavior point to novel effects.

Photodesorption measurements have been performed in a UHV chamber (base pressure $<5 \times 10^{-11}$ mbar) equipped with a quadrupole mass spectrometer (QMS) with a liquid nitrogen shroud and a sample preparation chamber with a LEED/Auger system and a QMS for temperature programmed desorption (TPD). Ag NPs were formed by depositing $\mathrm{Ag}$ atoms from an electron beam evaporator on an $\mathrm{Al}_{2} \mathrm{O}_{3} / \mathrm{NiAl}(110)$ substrate. The average size of the AgNPs was set at $8 \mathrm{~nm}$ as calibrated by 
separate STM observations [12]; their $(1,0)$ plasmon resonance is at $\sim 3.6 \mathrm{eV}[12,17]$. The sample was coolable to $20 \mathrm{~K}$ by liquid helium and could be dosed with Xe gas. The resulting multilayers of Xe were characterized by TPD. Annealing at $61 \mathrm{~K}$ formed a Xe monolayer on both $\mathrm{Al}_{2} \mathrm{O}_{3}$ and Ag NPs. Nanosecond light pulses ( $\sim 5$ ns wide, rep. rate $2 \mathrm{~Hz}$ ) from the second and third harmonics of a $\mathrm{Nd}$ :YAG laser (2.3 and $3.5 \mathrm{eV}, p$ - and $s$-polarized) and a $\mathrm{XeCl}$ excimer laser (4.0 eV, nonpolarized) were used for irradiation. The surface normal was oriented into the QMS axis, and the light was incident at 45 degrees. The desorption signals measured by the QMS were recorded simultaneously by two multichannel scalers with dwell times of $0.8 \mu \mathrm{s}$ and $5 \mathrm{~ms}$ triggered by the laser pulses to obtain time-of-flight (TOF) distributions (flight path of neutrals $\sim 19 \mathrm{~cm}$ ), and shot dependences of total photo-induced desorption (PID) yields of Xe, respectively.

Desorbing Xe atoms were detectable for fluences $(I)$ above $0.9 \mathrm{~mJ} / \mathrm{cm}^{2}$, for $p$-polarized light in the resonance, and about 5 times higher fluences off-resonance and for $s$-polarized light. Figure 1 shows TOF spectra of desorbing Xe, accumulated over 500 laser shots, for the parameters given in the caption. Trace (a), on-resonance $(h \nu=$ $3.5 \mathrm{eV}, p$-polarized, $I=1.1 \mathrm{~mJ} / \mathrm{cm}^{2}$ ), can be represented well by the sum of two shifted Maxwell-Boltzmann distributions corresponding to mean translational energies $\left\langle E_{t}\right\rangle=2 k T_{t}$ with $T_{t}=313 \mathrm{~K}$ for the main component, and a smaller contribution with $T_{t}=44 \mathrm{~K}$. Off resonance, e.g., for $h \nu=4.0 \mathrm{eV}$, unpolarized, a signal was barely

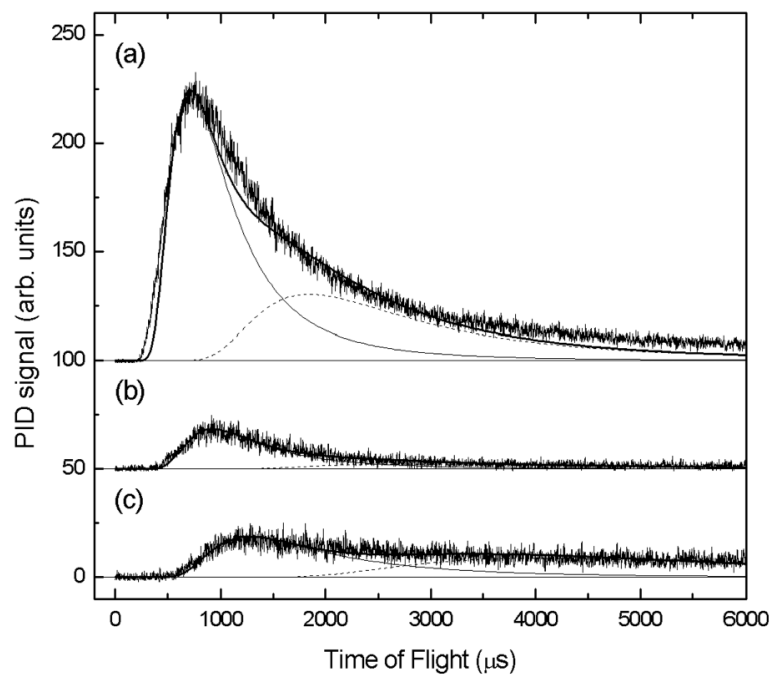

FIG. 1. Time-of-flight spectra of desorbing $\mathrm{Xe}$ atoms from (a) Xe/8-nm AgNP by excitation in the $(1,0)$ Mie resonance ( $h \nu=3.5 \mathrm{eV}, p$-pol., $1.1 \mathrm{~mJ} / \mathrm{cm}^{2}$ ), (b) Xe/8-nm AgNP by offresonance excitation $\left(h \nu=4.0 \mathrm{eV}\right.$, unpol., $\left.5.7 \mathrm{~mJ} / \mathrm{cm}^{2}\right)$, and (c) the bare alumina surface (excitation with $h \nu=3.5 \mathrm{eV}$, p-pol., $4.9 \mathrm{~mJ} / \mathrm{cm}^{2}$ ). The fit results by two shifted MaxwellBoltzmann distributions are shown as thick solid, thin solid, and dashed curves for the sum, the fast, and the slow components, respectively. Intensities of (b) and (c) are magnified by $\times 2$. detectable at $I=1 \mathrm{~mJ} / \mathrm{cm}^{2}$; at $5.7 \mathrm{~mJ} / \mathrm{cm}^{2}$ [Fig. 1(b)] the leading component has $T_{t}=161 \mathrm{~K}$, with some $20 \mathrm{~K}$ background. Similar results were obtained at $h \nu=2.3 \mathrm{eV}$ (not shown). Figure 1(c), Xe from the bare oxide without AgNPs, proves that the much stronger signals from the AgNP-covered surface indeed are induced by the particles. We estimate that the maximum temperature increase $(\Delta T)$ of the substrate surface at $I=5 \mathrm{~mJ} / \mathrm{cm}^{2}$ is about $60 \mathrm{~K}$, based on a laser-induced thermal desorption (LITD) [18] measurement of $\mathrm{Xe}\left(T_{t}=90 \mathrm{~K}\right)$ from a bare substrate at $30 \mathrm{~K}$. Thus $\Delta T$ is estimated as $12 \mathrm{~K}$ for $I=1 \mathrm{~mJ} / \mathrm{cm}^{2}$, as $\Delta T$ scales with $I$. Thermal heating of the AgNPs may be higher than that of the substrate in principle, but here it was not above $100 \mathrm{~K}$ at $1 \mathrm{~mJ} / \mathrm{cm}^{2}$, as confirmed by the absence of photodesorption of physisorbed $\mathrm{CO}$ and $\mathrm{N}_{2} \mathrm{O}$, which thermally desorb from the AgNPs at $60 \mathrm{~K}$ and $80 \mathrm{~K}$, respectively. The main conclusion then is that on resonance most of the desorption is nonthermal (termed hyperthermal subsequently), while off resonance at necessarily higher fluences the probable mechanism is thermal.

Measurement of the evolution of the Xe signals with the number of shots exhibited very unexpected behavior [Fig. 2(a)]. Off resonance at all fluences "normal" behavior was observed [Fig. 2(a)]: the Xe signal decreases roughly exponentially with the accumulated number of photons and is essentially gone after 300 shots. The noise on the signal is due to instabilities of laser and QMS. Similar behavior was seen on resonance at high fluences $\left(I>2 \mathrm{~mJ} / \mathrm{cm}^{2}\right.$; not shown). Drastically different behavior resulted on resonance for low fluences [Fig. 2(b), example shown: $1.8 \mathrm{~mJ} / \mathrm{cm}^{2}$. Groups of 500 shots were separated by pauses of $\sim 1 \mathrm{~min}]$. As seen, the signal does not decrease monotonically, but consists of a herd of spikes with strongly fluctuating intensities. When the laser beam is blocked during the pauses, the signal drops to the background level. A trend is seen that the heights of spikes seem to decrease by half after $\sim 2000$ shots, and the number of prominent spikes decreases with increasing number of shots. However, the height does not decrease monotonically and often even increases (in the case shown see the second bunch of laser shots where three large spikes are observed). It is noticeable that there is an offset at the bottoms of spikes which decays slowly. With higher laser fluences, this decaying offset became more significant, suggesting this offset is due to LITD. It was confirmed that the chaotic behavior is not due to the fluctuation of the laser intensity nor the inhomogeneity of the beam profile. The chaotic behavior is not observed in the case of photodesorption of NO from the AgNPs, for example, (there the TNI mechanism is operative).

To characterize the chaotic behavior shown in Fig. 2(b) we have calculated the Fourier transforms of the data. The insets in Fig. 2 show the resulting power spectra. In the normal case [Fig. 2(a)] the power spectrum has a broad decaying distribution. In contrast, for Fig. 2(b) only a flat 


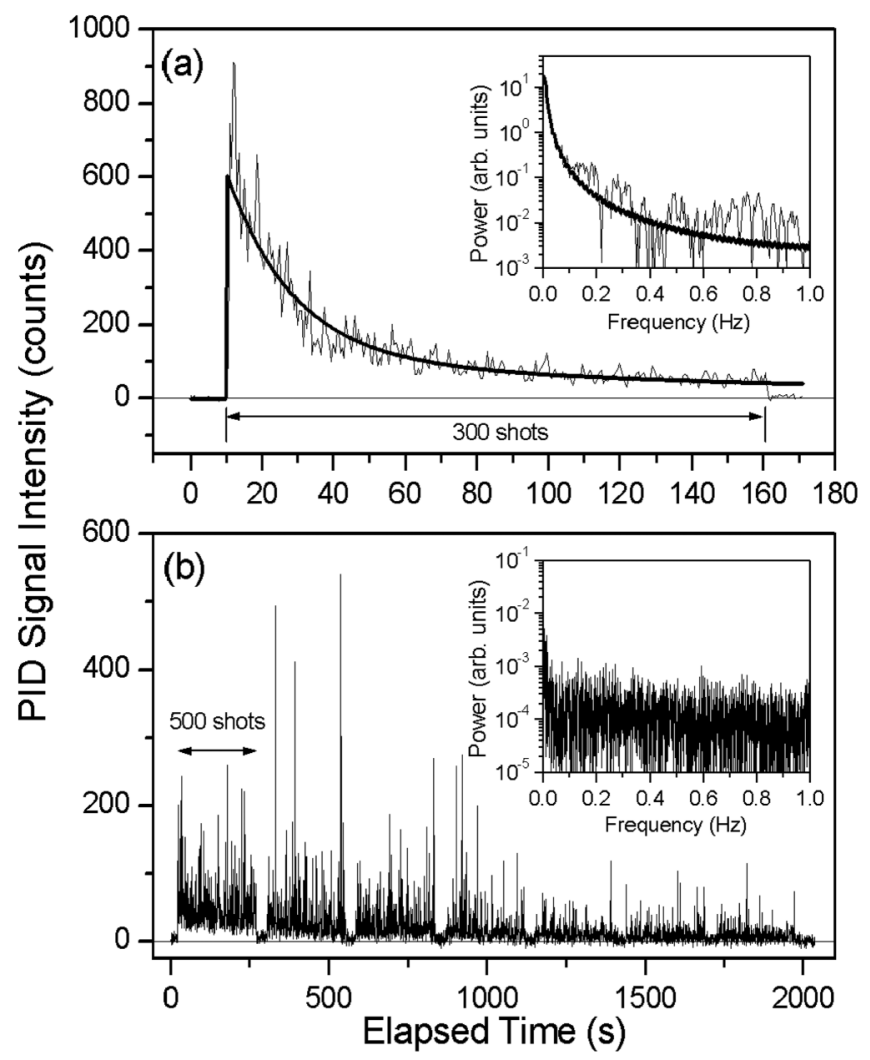

FIG. 2. Dependences of the Xe signal intensity on the total number of laser shots ( 2 shots per second), given as the time after starting the irradiation, for (a) excitation by $h \nu=2.3 \mathrm{eV}, p$-pol., $5.7 \mathrm{~mJ} / \mathrm{cm}^{2}$ for 300 continuous shots and (b) excitation by $h \nu=$ $3.5 \mathrm{eV}$, p-pol., $1.8 \mathrm{~mJ} / \mathrm{cm}^{2}$, with 1 -min pauses after each 500 shots. The numbers of laser shots are shown by arrows as guide. The insets show the power spectra of the data obtained by Fourier transformation.

spectrum is obtained. No peak, i.e., periodicity, can be recognized. Such a flat spectrum is a typical feature for chaotic systems [19].

Both the chaotic behavior and the hyperthermal energies of $\mathrm{Xe}$ were suppressed when multilayers of $\mathrm{Xe}$ were present, i.e., when the sample was not annealed to $61 \mathrm{~K}$ after dosing a thick Xe layer at low temperatures. This indicates that only Xe atoms in direct contact with the $\mathrm{Ag}$ NPs are responsible for the hyperthermal and chaotic desorption on resonance with the $(1,0)$ mode of the Mie plasmon (which lies at $h \nu=3.6 \mathrm{eV}, p$-polarization [17]) at low laser fluences. This suggests that the Mie plasmon plays a critical role in the hyperthermal chaotic desorption of Xe from Ag NPs.

The two outstanding and at least concurring (if not connected) features which we have to explain then are the hyperthermal desorption under resonant plasmon excitation, and its chaotic behavior. The latter property suggests that we have a case of plasmon coupling as derived by Stockman from calculations [20]. He has shown that the interaction of the plasmon fields of neighboring particles can lead to strong localization of excitation on a small number of particles ("hotspots"), which also leads to a drastic increase of the plasmon lifetime. While this effect is most obvious for fs excitation and fractal particle distributions, it does exist also for c.w. excitation. If a small part of our AgNP ensembles has the fitting properties, then such a strong but erratic removal of Xe for a comparatively small part of the total coverage is understandable. Fluctuations of the coupled fields then may introduce the chaotic behavior.

However, if we maintain that this type of desorption is hyperthermal, i.e., does not result from thermal heating of AgNPs but from an electronic action of the plasmon itself, a new mechanism of plasmon-induced desorption is needed as the usual mechanisms do not work here (see above). We propose that this is due to the modulation of the Pauli repulsion which defines the adsorption potential of Xe together with the van der Waals (vdW) attraction. To first approximation, the vdW attraction, which is established by the long-range dispersion force integrated over all $\mathrm{Ag}$ atoms of the AgNP, stays constant, whereas the Pauli repulsion, which stems from the short-range interaction between electrons of Xe and $s$-electrons spilling out from the AgNP surface, oscillates at the plasmon frequency. Under the plasmon excitation, the induced Pauli exclusion exerts a strong repulsive force on Xe towards the vacuum at a frequency of the plasmon ( $\sim 1 \mathrm{fs})$ which is much higher than the natural frequency of the ground state Xe (some ps). Although the impulse per plasmon cycle is small, Xe can be desorbed if it accumulates a sufficient momentum. A similar picture in terms of integrating over many small energy transfers has been used in the frictional or desorption induced by multiple electron transitions model [21] of electronically induced desorption; our model is very different in terms of the physical nature of the acting forces, however. Also, it is very different from the mechanism proposed [22] for the plasmon-mediated acceleration of (thermally evaporated) $\mathrm{Ag}^{+}$ions from $\mathrm{Ag}$ [5] by inverse bremsstrahlung.

In order to check the above mechanism, we performed molecular dynamics simulations. A potential in the BornMayer form $V(z)=A \exp (-K z)-\frac{C_{3}}{\left(z-z_{0}\right)^{3}}$ was used, where $z$ is the Xe-surface distance, $A$ and $K$ are parameters of Pauli repulsion, $C_{3}$ the dispersion coefficient, and $z_{0}$ the $\mathrm{vdW}$ reference plane position, with the values for Xe on $\operatorname{Ag}(111)$ [23]. The plasmon-modulated Pauli repulsion was implemented as a time-dependent displacement $\delta(t)=$ $\delta_{0} \sin \left(\frac{2 \pi t}{T}\right)$, where $\delta_{0}(>0)$ is the amplitude, $T$ the oscillation period. The displacement was included in the Pauli repulsion term as $V(z, t)=A \exp (-K(z-\delta(t)))-\frac{C_{3}}{\left(z-z_{0}\right)^{3}}$. The steeply repulsive part of the potential (we call it the "Pauli wall" because of its spatial coherence over the NP) moves back and forth by $\delta$ with time $t$, and largely coherently in space. The motion of a Xe atom on this 1dimensional potential was simulated by the velocity verlet method with $T=1.14 \mathrm{fs}$ (plasmon frequency at $3.6 \mathrm{eV}$ ). 
We find that Xe can desorb $\left(E_{t}>0\right)$ when $\delta_{0}>0.6 \AA$, which seems realistic for the plasmon-induced spread of electrons at silver surfaces [24]. $E_{t} / 2 k$ strongly depends on $\delta_{0}$ : With $\delta_{0}=0.68 \AA$ and $0.78 \AA, E_{t} / 2 k$ reaches $300 \mathrm{~K}$ and $1000 \mathrm{~K}$, respectively.

The above simulation showed that the Xe atom must collide with the "Pauli wall" 250 300 times before desorption. If the plasmon lifetime is only $10 \mathrm{fs}$ or less [10], giving only of the order of 10 cycles, then the accumulated energy should not suffice for most realistic parameter sets.

This last conclusion may establish a connection between the plasmonic coupling and the plasmon-induced desorption, via the required plasmon lifetime. Plasmonic coupling should lead to enhanced plasmon lifetimes [20], so the selective conditions of hotspot formation by coupling may at the same time enable the plasmon-induced desorption mechanism.

We realize that there are many rather strong approximations in this rough model, and many refinements are needed. For instance, the real process occurring is most likely not the desorption of individual Xe atoms. Rather, because of the lateral van der Waals attraction between the $\mathrm{Xe}$ atoms - which is not much smaller than that between $\mathrm{AgNP}$ and $\mathrm{Xe}$ - patches of Xe are more likely to desorb. This is compensated by the fact that the collective electron motion in the plasmon is in phase over the NP also. In fact, such a picture may give an additional explanation of the strong irregular bursts. Furthermore, the high field enhancements expected in the hotspots may lead to nonlinear effects which at present we cannot disentangle because of the chaotic behavior but which might be necessary to provide enough energy. On the experimental side, experiments with fs laser pulses, which should make it possible to obtain the time scale of the relevant processes directly, are clearly needed. We are tooling up for this at present.

In conclusion, we have observed hyperthermal, chaotic desorption of Xe from Xe monolayers on alumina deposited AgNPs by resonant excitation of the $(1,0)$ Mie plasmon with ns lasers. The chaotic behavior is observed at low laser fluences which do not cause thermal effects. It is most likely due to plasmonic coupling leading to hotspots of the electric field. Both unexpected characteristics are observed only for Xe monolayers and are quenched in multilayers; this shows that the hyperthermal energy of the desorbing Xe cannot be due to thermal heating of AgNPs, but must be connected to an electronic mechanism. Since Xe cannot desorb via transient negative ions, we suggest a new mechanism of plasmonic desorption by the accumulated momentum transfer of the repetitive Pauli repulsions between the collective motion of surface electrons and the Xe atom in its shallow physisorption well. A simple model calculation suggests that this mechanism can lead to sufficient energy transfer, but requires an interaction time longer than the plasmon lifetime of a single AgNP. As plas- monic coupling leads to an enhancement of plasmon lifetime, the two effects might well be intimately connected.

We thank Peter Feulner for very helpful suggestions and discussions, and Daniel Mulugeta and Walter Wachsmann for their contributions to the experiments. This work has been supported by the Deutsche Forschungsgemeinschaft within priority program SPP1093 (Dynamik von Elektronentransferprozessen an Grenzflächen), the German-Israeli Foundation (Dynamics of Electronic Processes in a Confined Environment), the Fonds der Chemischen Industrie, and the NEDO International Joint Research Grant on Photon and Electron Controlled Surface Processes.

[1] V. P. Zhdanov and B. Kasemo, J. Phys. Condens. Matter 16, 7131 (2004).

[2] K. Watanabe, D. Menzel, N. Nilius, and H.-J. Freund, Chem. Rev. 106, 4301 (2006).

[3] U. Kreibig and M. Vollmer, Optical Properties of Metal Clusters (Springer, Berlin, 1995).

[4] W. Hoheisel, K. Jungmann, M. Vollmer, R. Weidenauer, and F. Träger, Phys. Rev. Lett. 60, 1649 (1988).

[5] M. J. Shea and R. N. Compton, Phys. Rev. B 47, 9967 (1993).

[6] R. T. Kidd, D. Lennon, and S. R. Meech, J. Chem. Phys. 113, 8276 (2000), and earlier work cited therein.

[7] K. Wettergren, B. Kasemo, and D. Chakarov, Surf. Sci. 593, 235 (2005).

[8] S. Kwiet, D. E. Starr, A. Grujic, M. Wolf, and A. Hotzel, Appl. Phys. B 80, 115 (2005).

[9] F. M. Zimmermann and W. Ho, Surf. Sci. Rep. 22, 127 (1995).

[10] J. Bosbach, C. Hendrich, F. Stietz, T. Vartanyan, and F. Träger, Phys. Rev. Lett. 89, 257404 (2002).

[11] F. Stietz, Appl. Phys. A 72, 381 (2001).

[12] N. Nilius, N. Ernst, and H.-J. Freund, Phys. Rev. Lett. 84, 3994 (2000).

[13] C. M. Wong, J. D. McNeill, K. J. Gaffney, N.-H. Ge, A. D. Miller, S. H. Liu, and C. B. Harris, J. Phys. Chem. B 103, 282 (1999).

[14] D. Menzel and R. Gomer, J. Chem. Phys. 41, 3311 (1964).

[15] P. A. Redhead, Can. J. Phys. 42, 886 (1964).

[16] N. Schwentner, F.-J. Himpsel, V. Saile, M. Skibowski, W. Steinmann, and E. E. Koch, Phys. Rev. Lett. 34, 528 (1975).

[17] F. Evers, Ch. Rakete, K. Watanabe, D. Menzel, and H.-J. Freund, Surf. Sci. 593, 43 (2005).

[18] R. B. Hall, J. Phys. Chem. 91, 1007 (1987).

[19] H. G. Schuster, Deterministic Chaos: An Introduction (Physik-Verl., Weinheim, 1984).

[20] M. I. Stockman, Phys. Rev. Lett. 84, 1011 (2000).

[21] M. Brandbyge, P. Hedegård, T. F. Heinz, J. A. Misewich, and D. M. Newns, Phys. Rev. B 526042 (1995).

[22] R. H. Ritchie, J. R. Manson, and P. M. Echenique, Phys. Rev. B 49, 2963 (1994).

[23] E. Zaremba and W. Kohn, Phys. Rev. B 13, 2270 (1976).

[24] A. Liebsch, Phys. Rev. B 48, 11317 (1993). 